\title{
An Electro-mechanical Model of the Heart for Cardiac Image Analysis
}

\author{
M. Sermesant ${ }^{1}$, Y. Coudière $^{2}$, H. Delingette ${ }^{1}$, N. Ayache ${ }^{1}$, and J.A. Désidéri ${ }^{2}$ \\ 1 EPIDAure Research Project, INRIA Sophia Antipolis \\ 2 Sinus Research Project, INRIA Sophia Antipolis
}

\begin{abstract}
A simple electro-mechanical model of the heart is derived to best fit available cardiac images. The model is based on anisotropic linear elasticity and, from the electrical point of view, on the finite-element discretisation of the FitzHugh-Nagumo electric wave-propagation model. Present simulations include static image segmentation. By including biological and physical a priori knowledge, more realistic 4D images segmentation of the cardiac motion are expected.
\end{abstract}

\section{Introduction}

Cardiovascular pathologies are the first mortality cause in the industrialised countries. Estimating quantitative parameters of the ventricle function from images is a key to reach a better understanding of the cardiac motion but also to detect ischemic zones, to measure the pathology extent and to control the therapy effectiveness. These quantitative parameters, like the ejection fraction, myocardium thickness and local strain and stress constitute the ventricular cardiac function.

Some of these parameters can be efficiently extracted from the deformation of geometric surfaces [12. But these surfaces do not include any biological and physical a priori knowledge to guide their deformations where boundary data is missing [15, 16. Moreover, only the apparent motion (ie. displacement along the normal direction) can be reconstructed.

In this paper, we propose a framework to extract these parameters of interest from a simplified electro-mechanical model of the heart. Basically, unlike previous approaches, we have constructed an active model of the left and right ventricles; this model is activated by an electrical wave modifying its stiffness and shape and also constrained by the additional geometry originating from medical images. Although this is a work in progress, we believe that the proposed framework will allow a better understanding of the heart's behaviour.

\section{Geometric Model}

Our geometric model consists of a mesh of the whole myocardium (including both right and left ventricles) and of the myocardium fibre directions. Indeed, the fibres architecture has a great influence on the motion and on the propagation 
of the electrical excitation. It is based on data available from the Bioengineering Research Group of the University of Auckland, New Zealand [9, 13]. This data has been obtained by the dissection of a dog's heart and is composed of a mesh of 256 nodes and 180 hexahedra, and of a fibre direction at each node.

This hexahedral mesh presents the advantage of resulting from the deformation of a 3D regular grid. However, it contains tetrahedra of very different shape and size, and this may cause numerical difficulties. Instead, we are currently using tetrahedral meshes because this allows us to locally refine the mesh in parts of interest and to avoid the use of Gaussian quadrature to build stiffness matrices. In fact, different meshes with varying resolutions are built for the electrical and mechanical aspects of the computation.

To switch from hexahedral to tetrahedral meshes, we first triangulate the surface of the hexahedral mesh, and then refine it to the desired resolution while keeping sufficient quality triangles. Then a tetrahedral mesh is produced based on this triangulated surface and finally, the fibre directions are tri-linearly extrapolated and interpolated.

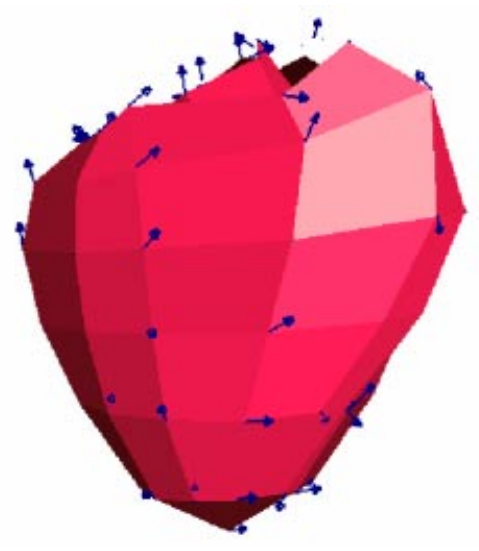

(a) Hexahedral Mesh

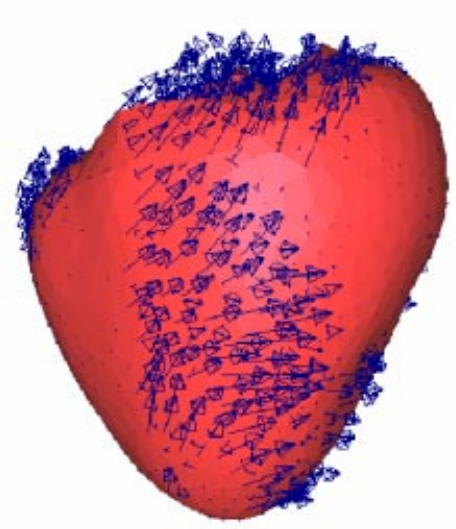

(b) Tetrahedral Mesh

Fig. 1. Myocardium meshes of different topologies, with the fibre directions

\section{Mechanical Model}

The myocardium is a nonlinear viscoelastic anisotropic active material [7, 6]. It is composed of fibre bundles spiralling around the two ventricles. Obviously, the physical model has to be simple enough for computational purposes. Therefore we are limiting ourselves to a linear anisotropic constitutive law. The internal stress-strain relationship in a frame related to the fibre direction is given by: 


$$
\sigma=\left(\begin{array}{cccccc}
A(\lambda+2 \mu) & \lambda & \lambda & 0 & 0 & 0 \\
\lambda & \lambda+2 \mu & \lambda & 0 & 0 & 0 \\
\lambda & \lambda & \lambda+2 \mu & 0 & 0 & 0 \\
0 & 0 & 0 & A \mu & 0 & 0 \\
0 & 0 & 0 & 0 & A \mu & 0 \\
0 & 0 & 0 & 0 & 0 & \mu
\end{array}\right) \varepsilon
$$

where $\varepsilon=\left(\operatorname{grad}(U)+\operatorname{grad}(U)^{t}\right) / 2$ with $U$ the displacement, $\lambda$ and $\mu$ are the Lamé constants and $A$ is a coefficient of anisotropy introduced in Hooke's law.

We have then a linear relationship between the internal body force and the displacement: $F=K U$ where $K$ is the stiffness matrix.

\section{Electrical Model}

Among the various models for the electric wave-propagation is the system of FitzHugh-Nagumo [4]:

$$
\begin{aligned}
& \frac{\partial u}{\partial t}=\Delta u+\lambda f(u)-z \\
& \frac{\partial z}{\partial t}=\epsilon(k u-z)
\end{aligned}
$$

where $f(u)=u(1-u)(u-a)$.

Here, $x=\bar{x} / L, t=\bar{t} / t_{\text {ref }}, u=\left(\bar{u}-u_{0}\right) / u_{\text {ref }}$ and $z$ are dimensionless variables. $\bar{u}$ is the actual transmembrane potential, $L$ is the size of the heart and $t_{\mathrm{ref}}=$ $L^{2} / D$ is a reference time ( $D$ the diffusion).

We are interested in this simple model since it correctly captures qualitative aspects of excitation and propagation, and has been successfully used in 3D computations [2, 19, 14, 5, 20].

Various modifications of the original FitzHugh-Nagumo equations have been proposed [8, 18, 11, 1, 10] to improve this model, in particular with respect to the shape of the action potential and the restitution properties (APD). We plan to incorporate one of these in our future work.

The theoretical aspects of (11) have been widely studied [21: a travelling wave of fixed shape and speed should appear or not, depending on the initial excitation being above or below a threshold.

In a first stage, solutions to (1) have been approximated by using a standard $P 1$ Lagrange finite element procedure (with mass lumping and first order numerical integration at vertices), on the given tetrahedral anatomical mesh. The Euler explicit time integration is performed to advance computations:

$$
\begin{aligned}
M \frac{u^{n+1}-u^{n}}{\Delta t} & =K u^{n}+M f\left(u^{n}\right)-M z^{n}, \\
\frac{z^{n+1}-z^{n}}{\Delta t} & =\epsilon\left(k u^{n}-z^{n}\right),
\end{aligned}
$$


where $u^{n}$ and $z^{n}$ are the vector of the nodal values of the approximates $u$ and $z$ at time $t^{n}=n \Delta t . K$ is a stiffness matrix and $M$ is the mass matrix (diagonal here).

In a second stage, anisotropic behaviour will be accounted for by letting the diffusion matrix $D$ depend on the local fibre orientation.

One remaining and crucial problem (of great importance) is that some appropriate boundary conditions must be imposed at the junctions between the special conduction system (Purkinje fibres) and the myocardium, which is not well known so far [5] 17. We have followed an approach which consists in assuming that the junctions region is located near the apex, below a plane that cuts the main heart axis.

Potential maps are presented at different time steps (Fig. 2), starting after a wave has been initiated at the apex and propagating while taking its complete shape.
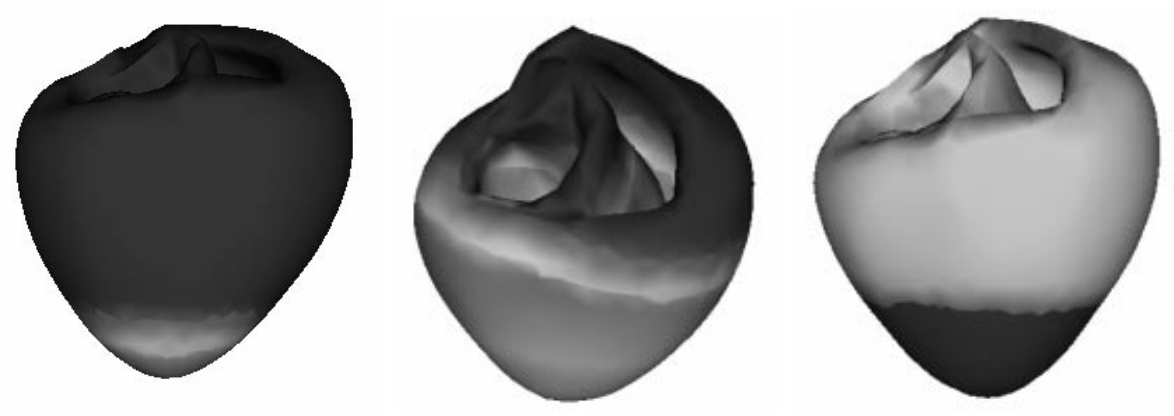

Fig. 2. Surface mapping of the $3 \mathrm{D}$ potential at different time steps

\section{Activation Model}

Each heart-beat cycle, a depolarization wave is initiated and propagates along the myocardium during about $10 \%$ of the total cardiac cycle. It induces a contraction of the whole myocardium, which produces a local stress tensor $\sigma_{a}=\alpha f \otimes f$, where $f$ is the fibre direction and $\alpha$ the activation rate, which is directly related to the parameter $u$ of Section 4 .

If $n$ is the external normal, by virtue of the Gauss theorem, the equivalent force is:

$$
\mathbf{F}_{a}=\int_{V} \operatorname{div}\left(\sigma_{a}\right) d v=\int_{V} \operatorname{div}(\alpha f \otimes f) d v=\int_{S}(\alpha f \otimes f) \bar{\otimes} n d s .
$$

Therefore, when a fibre is activated, its contraction is modelled as a pressure applied to the surface of the tetrahedron in the fibre direction.

Furthermore, in addition to the extra term $\sigma_{f}$, the electrical activation of the myocardium modifies its elastic properties. To simplify the model, we propose 

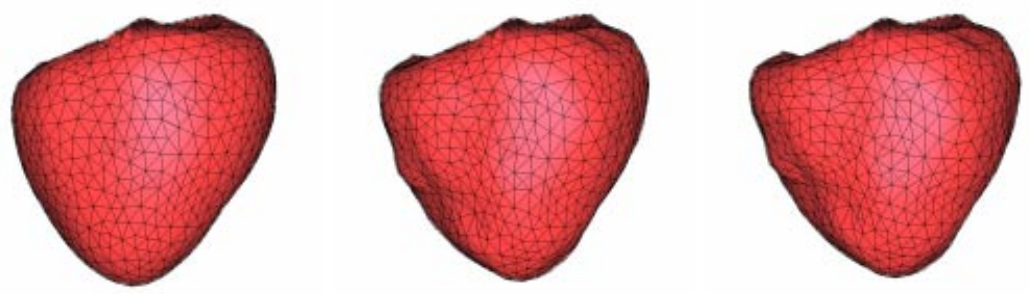

Fig. 3. Effect of the fibres contraction on the model.

to approximate the non-linear behaviour of the myocardium by a series of linear models that are only valid during a small part of the cardiac cycle.

\section{Interaction with Cardiac Images}

The deformation of our model is guided by $3 \mathrm{D}$ or $4 \mathrm{D}$ images. The material's anisotropy, through the constitutive law and the electrical activation, is employed to better recover the tangential motion of the ventricles.
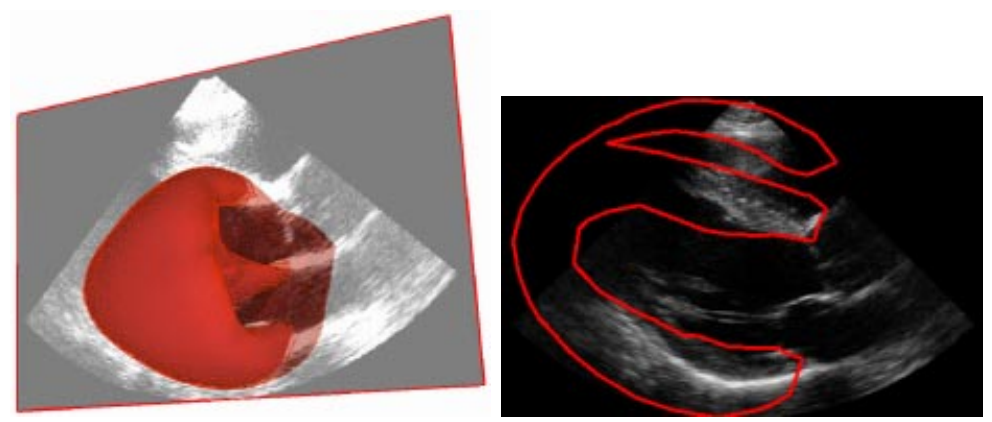

Fig. 4. 3D model in a slice of a 3D ultrasound image.

At time step $t$, for each node of the mesh surface, we look for the maximum gradient norm along the surface normal direction within a given distance range to find the closest boundary point of the image. We can also use a priori knowledge on the image: in ultrasound images, as we know that cardiac structures appear as grey areas on a black background, the gradient direction can help determine if the boundary point found is on the right side of the myocardium. Additionally, we can look for homogeneous areas in a given range of grey values (region approach).

Then, we apply a force $\mathbf{F}_{i}$ which is proportional to the distance to the closest boundary point of the image from the considered point of the mesh. 
We control the effect of those forces with two parameters:

- the proportionality factor to compute those forces from distances;

- the constitutive law of the material (Lamé constants), that controls the stress-strain relationship and the incompressibility;

For the initialisation, we use constants that allow the model to vary importantly, as we adapt the model to the patient heart. Subsequently, during the sequence, we fix the parameters to values found in the literature for the cardiac myocardium, which make it incompressible, for example. As rheological data is complicated to measure in-vivo, this procedure is also a way to adjust the Lamé constants. Furthermore, because of the volumetric nature of our model, it strongly decreases the importance of image's outliers in the motion estimation since it strongly constrains the geometric (for instance the thickness of the myocardium wall) and physical behaviour.

\section{Global Heart Model}

We use mass-lumping in a Newtonian differential equation with an explicit integration scheme to compute the position $\mathbf{P}$ of each vertex:

$$
\left(\frac{1}{\Delta t^{2}}-\frac{\gamma}{2 \Delta t}\right) \mathbf{P}^{t+1}=\mathbf{F}_{i}+\mathbf{F}_{a}+\mathbf{F}+\frac{2}{\Delta t^{2}} \mathbf{P}^{t}-\left(\frac{1}{\Delta t^{2}}+\frac{\gamma}{2 \Delta t}\right) \mathbf{P}^{t-1},
$$

with $\gamma$ the damping factor and $\mathbf{F}$ the internal forces computed from linear elasticity plus the boundary conditions (in particular the ventricular pressures). The model is activated through the forces $\mathbf{F}_{a}$ and constrained by the cardiac images through the external forces $\mathbf{F}_{i}$.

\section{Results and Perspectives}

This model is really appropriate for the segmentation of ultrasound data, which is sparse and noisy and where a priori knowledge is valuable.

The results (Fig. 5) show a rather good match between the mesh and the image data. The radial deformation which is only constrained by the stiffness of the material is however not so accurately adjusted. To improve this, further work on the elastic model and force adjustment is necessary.

In future work, the mechanical model will be improved to better account for normal strength and non linear laws of deformation. Additionally, a realistic simulation of the electric wave-propagation should include, besides the ventricles, the special conduction system (Purkinje Network). Particular efforts will be made in this direction also.

In practice, the model will be forced to fit $4 \mathrm{D}$ echocardiographic images sequences synchronised on the electrocardiogram: the electric wave will be deduced from the electrocardiographic measurements, then the activation state will be 

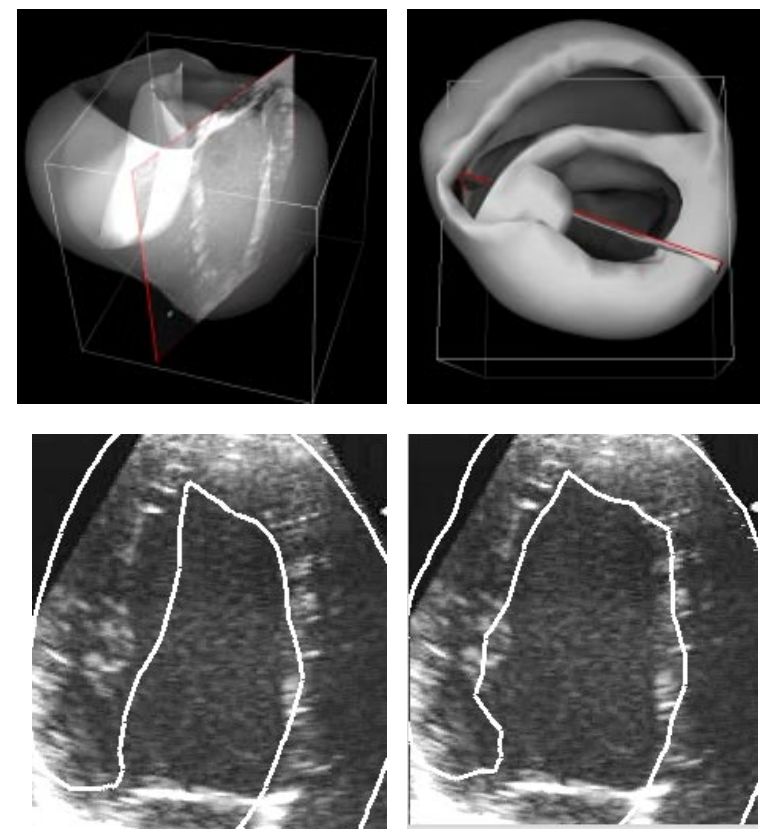

Fig. 5. (Top) 3D model in a slice of a 3D ultrasound image. (Bottom) Intersection of the model and the image, before (left) and after (right) deformation.

known at each time step. Hence, at each time step, the mechanical properties will be evaluated and the model will be deformed to fit the image1.

\section{Acknowledgements}

This work is a part of the multidisciplinary project ICEMA (standing for Images of the Cardiac Electro-Mechanical Activity) a collaborative research action between different INRIA projects which aim is to build a generic dynamic model of the beating heart and a procedure to automatically adjust the parameters to any specific patient [3].

\section{References}

[1] R. Aliev and A. Panfilov. A simple two-variable model of cardiac excitation. Chaos, Solitons and Fractals, 3(7):293-301, 1996.

[2] A.V. Holden AV and A.V. Panfilov. Computational biology of the heart, chapter Modelling propagation in excitable media, pages 65-99. John Wiley \& Sons, 1996.

[3] N. Ayache, D. Chapelle, F. Clément, Y. Coudière, H. Delingette, J.A. Désidéri, M. Sermesant, M. Sorine, and J. Urquiza. Towards model-based estimation of the

${ }^{1}$ Additional images and videos are available at http://www-sop.inria.fr/epidaure/personnel/Maxime.Sermesant/heartmodel/ 
cardiac electro-mechanical activity from ECG signals and ultrasound images. In Functional Imaging and Modeling of the Heart (FIMH'01), 2001 (submitted).

[4] A. L. Bardou, P. M. Auger, P. J. Birkui, and J.-L. Chassé. Modeling of cardiac electrophysiological mechanisms: From action potential genesis to its propagation in myocardium. Critical Reviews in Biomedical Engineering, 24:141-221, 1996.

[5] O. Berenfeld and J. Jalife. Purkinje-muscle rentry as a mechanism of polymorphic ventricular arrhytmias in a 3-dimensional model of the ventricles. Circ. Res., 82:1063-1077, 1998.

[6] J. Bestel, F. Clément, and M. Sorine. A biomechanical model of muscle contraction. In Medical Image Computing and Computer-Assisted intervention (MICCAI'01), 2001.

[7] Y. C. Fung. Biomechanics, Mechanical properties of living tissues. SpringerVerlag, 1993.

[8] J. Hindmarsh and R. Rose. A model of the nerve impulse using two first-order differential equations. Nature, (296):162-164, 1982.

[9] P. J. Hunter and B. H. Smaill. The analysis of cardiac function: a continuum approach. Biophysical molecular Biology, 1988.

[10] Z. Knudsen, A.V. Holden, and J. Brindley. Qualitative modelling of mechano electrical feedback in a ventricular cell. Bulletin of mathematical biology, 6(59):115$181,1997$.

[11] B. Kogan, W. Karplus, B. Billett, A. Pang, H. Karagueuzian, and S. Khan. The simplified fitzhugh-nagumo model with action potential duration restitution: effects on 2d wave propagation. Physica D, (50):327-340, 1991.

[12] J. Montagnat and H. Delingette. Space and time shape constrained deformable surfaces for 4D medical image segmentation. In Medical Image Computing and Computer-Assisted Intervention (MICCAI'00), 2000.

[13] M. Nash. Mechanics and Material Properties of the Heart using an Anatomically Accurate Mathematical Model. PhD thesis, University of Auckland, 1998.

[14] A. Panfilov and A. Holden. Computer-simulation of reentry sources in myocardium in 2 and 3 dimensions. Journal of Theoretical Biology, 3(161):271-285, 1993.

[15] X. Papademetris, A. J. Sinusas, D. P. Dione, and J. S. Duncan. Estimation of 3D left ventricle deformation from echocardiography. Medical Image Analysis, 5, 2001.

[16] Q.C. Pham, F. Vincent, P. Clarysse, P. Croisille, and I. Magnin. A FEM-based deformable model for the 3D segmentation and tracking of the heart in cardiac mri. In Image and Signal Processing and Analysis (ISPA'01), 2001.

[17] A.E. Pollard, N. Hooke, and C.S. Henriquez. Cardiac propagation simulation. Critical Reviews in biomedical Engineering, 20(3,4):171-210, 1992.

[18] J. Rinzel. Excitation dynamics: insights from simplified membrane models. Federation Proceedings, 15(44):2944-2946, 1985.

[19] J. Rogers, M. Courtemanche, and A. McCulloch. Computational biology of the heart, chapter Finite element methods for modelling impulse propagation in the heart, pages 217-233. John Wiley \& Sons, 1996.

[20] K. Simelius, J. Nenonen, R. Hren, and B.M. Horacek. Electromagnetic extracardiac fields simulated with a bidomain propagation model. In J. Nenonen, R.J. Ilmoniemi, and T. Katila, editors, International Conference on Biomagnetism (Biomag'00), 2000.

[21] J. Smoller. Shock Waves and Reaction-Diffusion Equations. Springer-Verlag (Grundlehren der mathematischen Wissenschaften 258), 1983. 